\title{
Modified Fowler-Milne method for the spectroscopic determination of thermal plasma temperature without the measurement of continuum radiation
}

\author{
Shuiliang Ma, ${ }^{1,2, a)}$ Hongming Gao, ${ }^{1}$ and Lin $\mathrm{Wu}^{1}$ \\ ${ }^{1}$ State Key Laboratory of Advanced Welding Production Technology, Harbin Institute of Technology, \\ Harbin 150001, People's Republic of China \\ ${ }^{2}$ Plasma Research Laboratory, Australian National University, Canberra ACT 0200, Australia
}

(Received 6 November 2010; accepted 23 November 2010; published online 24 January 2011)

\begin{abstract}
A technique based on the Fowler-Milne method for the spectroscopic determination of thermal plasma temperatures without measuring continuum radiation is presented. This technique avoids the influence of continuum radiation with the combined line and continuum emission coefficients to derive the plasma temperatures. The amount of continuum emission coefficient is estimated by using an expression related to the Biberman factors. Parameters that affect the accuracy of the proposed technique and errors in the measured plasma temperatures are analyzed. It is shown that, by using the Ar I $696.5 \mathrm{~nm}$ line with a bandwidth of $3.27 \mathrm{~nm}$ without taking into account the continuum radiation, the plasma temperature measured will be lower on the order of up to 1000-3000 K for temperatures from 20000 to $24000 \mathrm{~K}$. The theoretically predicted temperature errors are in good agreement with the experimental results, indicating that the proposed technique is reliable for plasma temperature measurement. (C) 2011 American Institute of Physics. [doi:10.1063/1.3529019]
\end{abstract}

\section{INTRODUCTION}

Thermal plasmas characterized with high temperature and high power density are stable and can be operated with relatively low equipment cost. Due to these merits, they are widely used in many industrial areas, such as welding, cutting, spraying, surface modification, and synthesis of fine powders. ${ }^{1}$ In order to improve the understanding and performance of these applications the plasma key parameters, such as the electron temperature and density, should be measured.

There are many methods for the measurement of thermal plasma parameters. The Langmuir probe is a simple and flexible diagnostic tool, but the probe will disturb the parameter distribution of the source. Furthermore, the interpretation of the data produced is difficult because no simple, comprehensive underlying theory exists for high temperature thermal plasmas. ${ }^{2}$ Another method is the active noninvasive spectroscopic diagnostic technique with the Thomson scatting as a typical example. In this case, although the results are usually easy to interpret, the experimental setup is expensive and the method is not easy. ${ }^{3}$ Consequently, optical emission spectroscopy comes to be one of the most extensively applied diagnostic methods for the study of thermal plasmas. From measured spectroscopic data, the plasma temperature can be determined by several different methods, such as using the atomic line intensities or intensity ratios of two or more spectral lines, and the ratios of line-to-continuum intensity. ${ }^{4}$ Among these methods, the Fowler-Milne method ${ }^{5}$ has been widely used due to its precision and stability. ${ }^{6-8}$

Considering the large gradients in the thermal plasma sources, to characterize their properties with spectroscopic methods spatially resolved plasma intensities should be

a)Electronic mail: shlgma@126.com. measured. This can be realized by two different techniques. One technique using the point-by-point scanning approach with a spectrograph is relatively simple and accurate, but the long time required to finish the scanning limits its application only to steady state plasmas. ${ }^{5-8}$ The other one based on monochromatic imaging with narrow-band filters in conjunction with a detector is a more efficient approach and has attracted great interest in recent years due to its very short acquisition time. This technique has actually been used for the diagnostics of different kinds of plasmas. For example, use a line ratio imaging method to reveal the cause of the enhanced glow in the void of the dust particle cloud ${ }^{9}$ and to determine the temperature and metal vapor concentration in a copper breaking arc. ${ }^{10}$

In a recent study, ${ }^{11}$ we presented the diagnostics of a static free-burning arc based on the combination of the imaging technique and the Fowler-Milne method. The determined plasma temperatures are in quantitatively agreement with other measurements by a spectrograph. With this combined technique, we also characterized the properties of the freeburning arc in the stage after ignition. ${ }^{12}$ The imaging based spectroscopic technique has proved to be a reliable and suitable method for the diagnostics of arc plasmas in a steady or dynamic state. The drawback of this technique is that the measured plasma temperature will be a little lower especially for temperatures higher than $16000 \mathrm{~K}$, since the acquired arc intensity consists of both the spectral line intensity and the continuum radiation. For the imaging technique, it is possible to measure the continuum radiation with another narrow-band filter, but this will complicate the experiments. Therefore, other spectroscopic methods for the determination of plasma temperatures, which can avoid the influence of the continuum radiation without extra measurements, will be more attractive. 
This paper extends the Fowler-Milne method for the measurement of thermal plasma temperatures considering the influence of continuum radiation. To determine the amount of continuum radiation, the temperature-averaged Biberman factors are used. With the correctly estimated Biberman factor values, influence of the continuum radiation on the calculated plasma temperatures can almost be completely avoided. The proposed technique is particularly suitable for the diagnostics of dynamic plasmas with a monochromatic imaging system.

In Sec. II we describe the line and continuum radiation theory of a thermal plasma and the temperature measurement techniques based on the Fowler-Milne method. Section III treats the application of the modified technique to a freeburning argon arc. Possible errors of the new technique due to continuum radiation are discussed in Sec. IV. Conclusions are given in Sec. V.

\section{TEMPERATURE MEASUREMENT METHOD}

\section{A. Line and continuum radiation}

The light emitted from a thermal plasma consists of spectral lines and continuum radiation. These radiations are produced due to the excitation and deactivation of emitting atoms and ions and due to their interactions with free electrons, thus contain useful information of the conditions in the plasma source.

For an optically thin plasma in local thermodynamic equilibrium (LTE), the emission coefficient of an atomic spectral line corresponding to a transition from a level $m$ to a lower level $n$ is given by the following expression:

$$
\varepsilon_{1}=\frac{h c}{4 \pi \lambda} g_{m} A_{n m} \frac{n_{j}}{U_{j}} \exp \left(-\frac{E_{m}}{k T}\right),
$$

where $c, h$, and $k$ are, respectively, the speed of light, Planck's constant, and Boltzmann's constant, $\lambda$ is the wavelength of the spectral line, $A_{n m}$ is the transition probability, $g_{m}$ and $E_{m}$ are, respectively, the statistical weight and energy of the upper level, $n_{j}$ and $U_{j}$ are the number density and the partition function of the species $j$.

The continuum radiation of plasmas containing only rare gases is composed of the free-free (bremsstrahlung) and the free-bound (recombination radiation) emissions. The total emission coefficient of continuum radiation may be described by ${ }^{13-16}$

$$
\varepsilon_{\mathrm{c}}=\varepsilon_{\mathrm{ea}}^{\mathrm{ff}}+\varepsilon_{\mathrm{ei}}^{\mathrm{ff}}+\varepsilon_{\mathrm{ei}}^{\mathrm{fb}},
$$

at the right-hand side the first term is the contribution of the bremsstrahlung radiated from the electron-atom collisions, and the second and third terms are, respectively, the bremsstrahlung and the recombination radiation generated by the collision between electrons and ions. For the quantitative approximation of the three contributions, we refer to ${ }^{13-15}$

$$
\begin{aligned}
\varepsilon_{\mathrm{ea}}^{\mathrm{ff}}= & C_{\mathrm{ea}} Q\left(T_{\mathrm{e}}\right) \frac{n_{\mathrm{e}} n_{0}}{\lambda^{2}} T_{\mathrm{e}}^{3 / 2} \\
& \times\left[1+\left(1+\frac{h c}{\lambda k T_{\mathrm{e}}}\right)^{2}\right] \exp \left(-\frac{h c}{\lambda k T_{\mathrm{e}}}\right),
\end{aligned}
$$

$$
\begin{aligned}
\varepsilon_{\mathrm{ei}}^{\mathrm{ff}}= & \sum_{i} C_{\mathrm{ei}} Z_{i}^{2} \frac{n_{\mathrm{e}} n_{i}}{\lambda^{2} \sqrt{T_{\mathrm{e}}}} \exp \left(-\frac{h c}{\lambda k T_{\mathrm{e}}}\right) \xi_{i}^{\mathrm{ff}}\left(\lambda, T_{\mathrm{e}}\right), \\
\varepsilon_{\mathrm{ei}}^{\mathrm{fb}}= & \sum_{i} C_{\mathrm{ei}} Z_{i}^{2} \frac{n_{\mathrm{e}} n_{i}}{\lambda^{2} \sqrt{T_{\mathrm{e}}}}\left[1-\exp \left(-\frac{h c}{\lambda k T_{\mathrm{e}}}\right)\right] \\
& \times \frac{g_{i, 1}}{U_{i}} \xi_{i}^{\mathrm{fb}}\left(\lambda, T_{\mathrm{e}}\right),
\end{aligned}
$$

where $C_{\text {ea }}=1.026 \times 10^{-34} \mathrm{Jm}^{2} \mathrm{~K}^{-3 / 2} \mathrm{~s}^{-1} \mathrm{sr}^{-1}, C_{\text {ei }}=1.632$ $\times 10^{-43} \mathrm{Jm}^{4} \mathrm{~K}^{1 / 2} \mathrm{~s}^{-1} \mathrm{sr}^{-1}, Q\left(T_{\mathrm{e}}\right)$ is the average cross section for momentum transfer of electron-atom interactions, ${ }^{17} Z_{i}$ is the charge number of the ion, $T_{\mathrm{e}}$ is the electron temperature, $n_{0}, n_{i}$, and $n_{\mathrm{e}}$ are respectively, densities of atoms, ions, and electrons, $\xi_{i}^{\mathrm{ff}}$ and $\xi_{i}^{\mathrm{fb}}$ are the so-called free-free and freebound Biberman factors, which are functions of both wavelength and temperature and depend on the ionization degree, $g_{i, 1}$ is the statistical weight of the parent ion and $U_{i}$ is the partition function, $g_{i, 1} \simeq U_{i}$, because for argon plasmas the excited levels are at much higher energies relative to the ion ground state and thus excited level densities contribute not much to the partition function even in equilibrium. ${ }^{14,18}$ It is reported that in an argon plasma the amount of radiation emitted from the electron-atom collisions is less than $1 \%$ for temperatures above $8000 \mathrm{~K}$, and the free-free and free-bound radiations emitted from interactions of electrons with doubly ionized gases become important for temperatures higher than $18000 \mathrm{~K}^{15}$ Therefore, for a high-current free-burning argon arc, which is characterized with a temperature ranging up to more than $20000 \mathrm{~K}$, the latter should be considered. Substituting Eqs. (4) and (5) into (2) and ignoring the contribution of the electron-atom radiation, one obtains

$$
\varepsilon_{\mathrm{c}}=\varepsilon_{\mathrm{c} 1}+\varepsilon_{\mathrm{c} 2}=C_{\mathrm{ei}} \frac{n_{\mathrm{e}}}{\lambda^{2} \sqrt{T_{\mathrm{e}}}} \sum_{i=1}^{2} n_{i} Z_{i}^{2} \xi_{i}\left(\lambda, T_{\mathrm{e}}\right),
$$

where $\varepsilon_{\mathrm{c} 1}$ and $\varepsilon_{\mathrm{c} 2}$ are, respectively, the radiations emitted by collisions between electrons and the singly and doubly ionized atoms, and $\xi_{i}$ is the total Biberman factor, which is defined as

$$
\begin{aligned}
\xi_{i}\left(\lambda, T_{\mathrm{e}}\right)= & \exp \left(-\frac{h c}{\lambda k T_{\mathrm{e}}}\right) \xi_{i}^{\mathrm{ff}}\left(\lambda, T_{\mathrm{e}}\right) \\
& +\left[1-\exp \left(-\frac{h c}{\lambda k T_{\mathrm{e}}}\right)\right] \xi_{i}^{\mathrm{fb}}\left(\lambda, T_{\mathrm{e}}\right) .
\end{aligned}
$$

The Biberman factors are dimensionless correction parameters that account for the deviation from the hydrogenlike structure. The free-free Biberman factor is reported to be a very weak function of both wavelength and temperature, ${ }^{19}$ while the free-bound Biberman factor is dependent on both wavelength and temperature, especially for short wavelengths $(<450 \mathrm{~nm})$. For long wavelengths $(>800 \mathrm{~nm})$ the Biberman factors are usually close to a constant, since the difference between the high levels of different atoms becomes smaller. Hofsaess ${ }^{20}$ has calculated the Biberman factors at different wavelengths and temperatures for argon and some other gases, which are consistent with many experimental values. ${ }^{13}$ For relatively long wavelengths, however, the calculated data are always lower compared with 
experimentally measured values. The recent calculation by D'yachkov et al. ${ }^{21}$ which takes into account the plasma effects in the near-threshold region, does not show the sharp edge of the photoionization continuum and is in good agreement with experimental values.

Figure 1 shows the total Biberman factors for both singly and doubly ionized argon atoms as a function of wavelength and temperature, $\xi_{1}^{\mathrm{ff}}=1.23^{19}, \xi_{2}^{\mathrm{ff}}=1$, and the $\xi_{i}^{\mathrm{fb}}$ data are taken from the calculations of Hofsaess. (Some of the Hofsaess data can be found in Ref. 20. Additional Hofsaess calculations are derived from Refs. 22 and 23.) In Fig. 1(a) the theoretical results from D'yachkov et al. ${ }^{21}$ are also shown. The experimental values of the Biberman factors for doubly ionized gases are in scarcity. ${ }^{24}$ In Fig. 1(b) the only experimental data (to the knowledge of the authors) at $468.8 \mathrm{~nm}$ (Ref. 15) are compared with the calculation. Since the calculated values are much lower than the experimental data, this is also true for $\xi_{1}$, we expect that the actual values for $\xi_{2}^{\mathrm{fb}}$ should be larger. By enlarging the values of $\xi_{2}^{\mathrm{fb}}$ by a factor 2 , the obtained $\xi_{2}$ data are in agreement with the experimental measurements.

\section{B. The Fowler-Milne method}

In order to compute the theoretical emission coefficients needed to determine plasma temperatures, the plasma composition should be calculated. If the plasma is in LTE state and at constant pressure, the dependence of the particle densities on temperature can be obtained based on Dalton's law, the quasineutrality condition, and Saha equations. ${ }^{5}$ For freeburning arcs in argon, except the regions near the electrodes and the fringes of the arc, the central regions of the arc column with a plasma temperature higher than $12800 \mathrm{~K}$ are confirmed in LTE (Ref. 6) from both experimental measurements ${ }^{3,25}$ and theoretical analysis. ${ }^{26}$ With the computed plasma composition, the theoretical emission coefficient of the Ar I $696.5 \mathrm{~nm}$ line as a function of temperature was calculated using Eq. (1), as shown in Fig. 2. The calculated curve passes through a peak value at a temperature of about $15200 \mathrm{~K}$, the normal temperature of the atomic line. Provided that the plasma axial temperature is higher than the normal temperature, the measured radial emission coefficients will exhibit a corresponding off-axis maximum. Thus, assigning the normal temperature to the position of the maximum, the plasma temperatures at other radial positions can be derived. This is the so-called Fowler-Milne method, also known as the normal temperature method. ${ }^{5}$ The FowlerMilne method can provide more accurate results compared to other spectroscopic methods since the value of the transition probability, which has a large uncertainty, is not needed. Another merit of this method is that the absolute calibration of the plasma intensities is not necessary. In addition, the method gives quite accurate temperature values even when applied to strongly self-absorption atomic lines. ${ }^{27}$ Because of these advantages, this method has been widely used to derive radial temperature distributions in free-burning arcs from measured relative emission coefficients of atomic lines.

In the case of continuum radiation, the emission coefficient as a function of temperature can be calculated in an
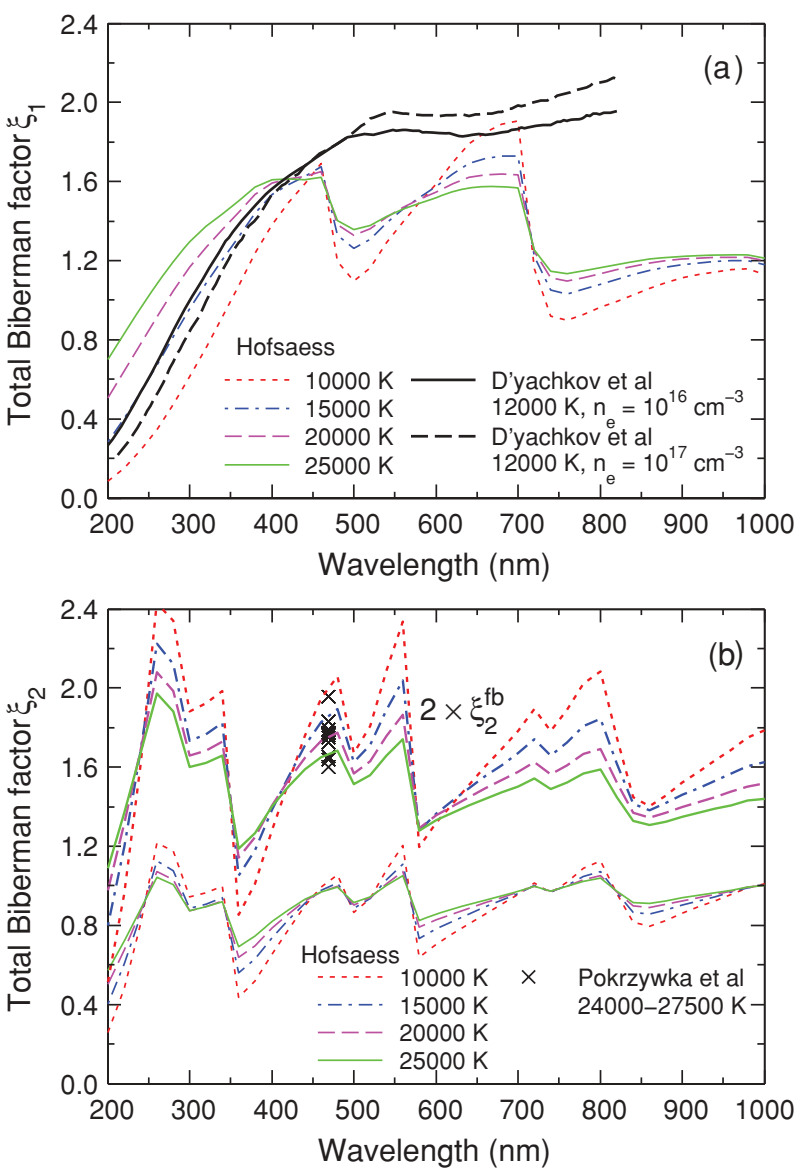

FIG. 1. (Color online) Total Biberman factors $\xi_{1}$ and $\xi_{2}$ for argon at different plasma temperatures as a function of wavelength computed with $\xi_{1}^{\mathrm{ff}}=1.23$, $\xi_{2}^{\mathrm{ff}}=1.0$, and the free-bound Biberman factor data taken from theoretical calculation by Hofsaess (Refs. 20,22, and 23). The recent calculation for $\xi_{1}$ by D'yachkov et al. (Ref. 21) and the experimental values for $\xi_{2}$ measured by Pokrzywka et al. (Ref. 15) are also presented.

analogous manner. From Fig. 1 it is easy to see that for temperatures between 15000 and $25000 \mathrm{~K}$, the values of $\xi_{1}$ and $\xi_{2}$ are only weakly dependent on temperature and thus can be assumed to be constants near $696.5 \mathrm{~nm}$. The emission coefficient curve of the continuum radiation at $696.5 \mathrm{~nm}$ calculated using Eq. (6) with $\xi_{1}=1.9$ and $\xi_{2}=1.6$ is shown in Fig. 2. Since at about $16500 \mathrm{~K}$ the curve shows a maximum, the plasma temperatures can also be derived from the measured radial profiles of the continuum emission coefficients. However, this gives doubtful temperatures due to several reasons. First, the lack of reliable data for $\xi_{2}$ will lead to large uncertainties in the derived results, especially for temperatures higher than $18000 \mathrm{~K}$. Second, the continuum emission coefficient curve for temperatures higher than $16500 \mathrm{~K}$ has small variations, which is difficult to be reconstructed reliably by Abel inversion and will cause large errors. Finally, the continuum radiation is probably to be affected by the broadening of the near spectral lines and has a low signal-to-noise ratio due to the much weaker intensity compared to that of the atomic lines. The continuum radiation is, therefore, not suitable for the determination of plasma temperatures by using the Fowler-Milne method though the measurement of intensity distributions at only one wavelength is needed. 


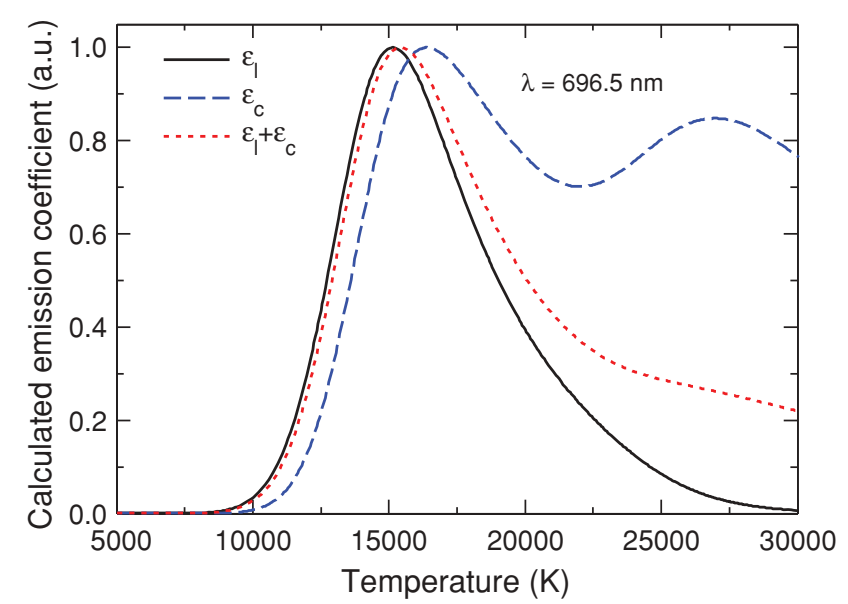

FIG. 2. (Color online) Dependence on temperature of the calculated emission coefficients for the Ar I $696.5 \mathrm{~nm}$ line, the continuum radiation at the same wavelength, and the total radiation with the continuum emission coefficient integrated over a wavelength interval of $3.27 \mathrm{~nm}$. All the curves have been normalized to their respective maximum values.

\section{The proposed technique}

The measured spectral line intensity unavoidably contains the continuum radiation near the same wavelength. If the continuum radiation cannot be measured separately and subtracted from the total intensity, the plasma temperatures derived from the measured total emission coefficients by applying the Fowler-Milne method will be affected. Figure 2 also shows the calculated total emission coefficient as a function of temperature, the continuum radiation was integrated over a wavelength range of $3.27 \mathrm{~nm}$. Compared with the normalized line emission coefficient, the normalized total emission coefficient is lower and higher, respectively, for temperatures below and above $15200 \mathrm{~K}$. Therefore, the calculated plasma temperature will be lower than that it should be when ignoring the continuum radiation.

To take into account the effect of the continuum radiation, we propose to calculate the plasma temperatures by using the emission coefficient curve of the total radiation instead that of the spectral line. Since the measured emission coefficient is also the combination of the line and continuum radiation, theoretically there will be no systematic errors in the determined temperatures.

For this modified temperature determination technique, the most significant advantage is that the measurement of the continuum radiation is not needed, which will be important in some cases in which the simultaneous measurement of the line and continuum radiation is not convenient. For example, when using narrow-band filters to acquire the plasma intensities, the measurement setup can be considerably simplified if only the distribution of the total emission coefficients is needed. Besides preserving all the advantages of the FowlerMilne method, the proposed technique even improves some of them. For example, the accessible temperature range for the Fowler-Milne method is about $10000-27000 \mathrm{~K}$, while for the new technique the range extends to above $30000 \mathrm{~K}$.

The possible limitation of the proposed technique, except those also for the Fowler-Milne method, is that the lack of accurate values for the Biberman factors $\xi_{1}$ and $\xi_{2}$ will cause some uncertainties for temperatures higher than $16000 \mathrm{~K}$. In most cases, the uncertainty will be relatively small since the amount of continuum radiation for temperatures not high enough is low compared to the total plasma radiation.

\section{EXPERIMENT}

\section{A. Experimental setup and measurement}

In the experiment, a direct current power source and a common tungsten inert gas welding torch were used to generate the arc. The torch was water cooled and has a ceramic nozzle with an internal diameter of $10 \mathrm{~mm}$. Argon (with purity of $99.9 \%$ ) was used as the shielding gas with a flow rate of $10 \mathrm{l} / \mathrm{min}$. The cathode electrode was a $3.2 \mathrm{~mm}$ diameter, tungsten rod ( $2 \% \mathrm{Ce}_{2} \mathrm{O}_{3}$ by mass) ground to a conical tip with an included angle $60^{\circ}$, and the anode electrode was a watercooled copper plate situated $5 \mathrm{~mm}$ below the cathode tip. The arc was started by a short high-voltage pulse and free burning at atmospheric pressure between the two electrodes.

Radiation from the arc was imaged by a lens system onto an image plane at a magnification of 2:1. A light aperture was employed to limit light from the arc for obtaining adequate spatial resolution. On the image plane, an optical fiber was mounted on a motor-driven table which can be moved in the horizontal and vertical directions. The internal diameter of the optical fiber was $0.2 \mathrm{~mm}$ and thus the scanning step along the horizontal direction was determined as $0.2 \mathrm{~mm}$. Spectral intensities from several layers of the arc were scanned with an observation distance at the arc of $0.25 \mathrm{~mm}$ in the vertical direction.

Light along different chords of the arc was transmitted by the optical fiber to the entrance slit of a $500 \mathrm{~mm}$ focal length spectrograph (Acton SP500i). In the spectrograph, a 300 grooves $/ \mathrm{mm}$ grating with a resolution of $0.156 \mathrm{~nm}$ was adopted for spectra dispersion. At the exit slit of the spectrograph spectra were detected by a charge-coupled device (Princeton TE/CCD-1100PF, $1100 \times 330$ pixels), which has a spectral range from 200 to $1000 \mathrm{~nm}$. When the 300 grooves $/ \mathrm{mm}$ grating was used, spectra within $170 \mathrm{~nm}$ could be acquired by the detector at one acquisition. Figure 3 shows the Ar I spectra between 680 and $860 \mathrm{~nm}$ measured at $1 \mathrm{~mm}$ below the arc cathode tip.

\section{B. Results}

The Ar I $696.5 \mathrm{~nm}$ line and the continuum radiation at a wavelength of $690.7 \mathrm{~nm}$ at different axial positions of the arc were scanned and recorded. We use this spectral line because it has a strong intensity, is well separated from other lines, and almost free from self-absorption. The measured intensity of the atomic spectral line at each chordal position was corrected by subtracting the corresponding continuum radiation intensity. The side-on measured spectral line intensity profiles were symmetrized, noise filtered, and Abel inverted using a modified discrete form ${ }^{28}$ of the Fourier-Hankel transform method to reconstruct the radial plasma emission coefficients.

Figure 4 shows the lateral plasma intensities measured $1 \mathrm{~mm}$ from the cathode tip for the Ar I $696.5 \mathrm{~nm}$ line 


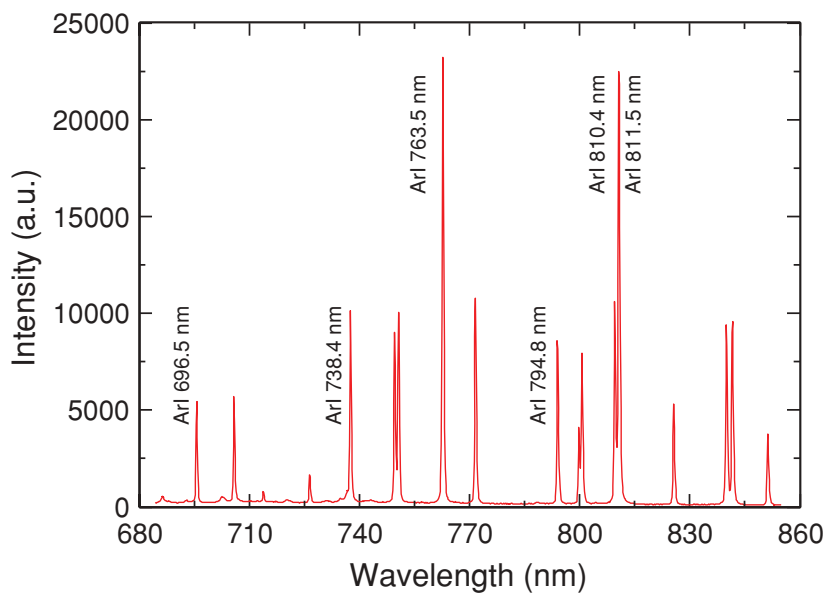

FIG. 3. (Color online) Emission spectrum between 680 and $860 \mathrm{~nm}$ of a 200 A free-burning argon arc measured at $1 \mathrm{~mm}$ below the cathode tip. The spectrum is dominated by the argon atomic line emission.

and the continuum radiation at $690.7 \mathrm{~nm}$ integrated over a wavelength interval of $3.27 \mathrm{~nm}$. The intensity profile for the Ar I $696.5 \mathrm{~nm}$ line was obtained by subtracting the continuum radiation from the measured total intensities (the raw data). Both the processed line and total intensity profiles are also presented. Figure 5(a) shows the corresponding reconstructed radial distributions of the normalized plasma emission coefficients for the spectral line with and without subtracting the continuum radiation. Each profile of the radial emission coefficients shows an off-axis maximum. Within and outside the maximum position the total emission coefficient is, respectively, larger and smaller than that of the spectral line.

Figure 5(b) shows the radial dependence of the plasma temperatures calculated from the line and total emission coefficients by using the Fowler-Milne method. It is clear that at any radial position of the arc, the determined temperature without correction (derived from the sum of line and continuum emission coefficients) is a little lower compared with that taking into account the effect of continuum radiation; the discrepancy becomes larger with the increase of the plasma tem-

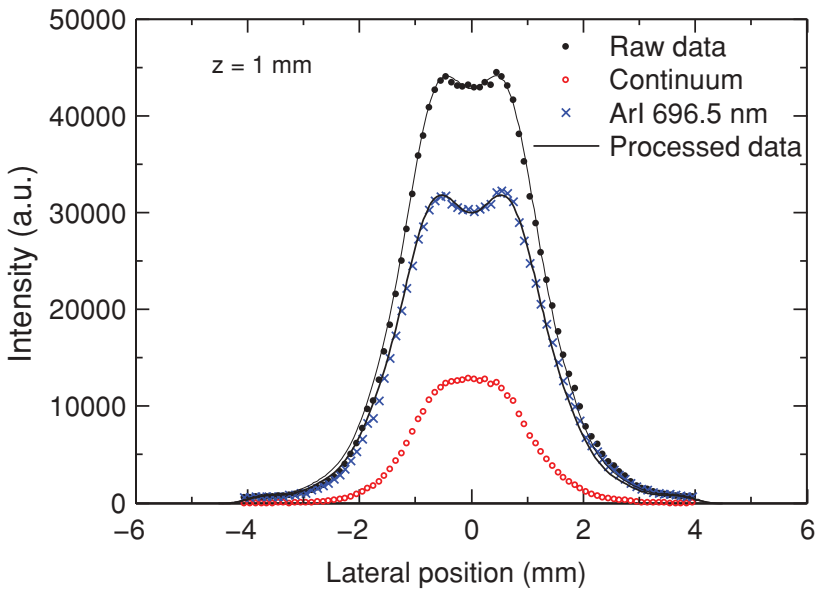

FIG. 4. (Color online) Lateral variation of the measured plasma intensities for the Ar I $696.5 \mathrm{~nm}$ spectral line, the continuum radiation at $690.7 \mathrm{~nm}$ integrated over a wavelength interval of $3.27 \mathrm{~nm}$, their sum (the raw data), and the data after symmetrization and noise filtering.
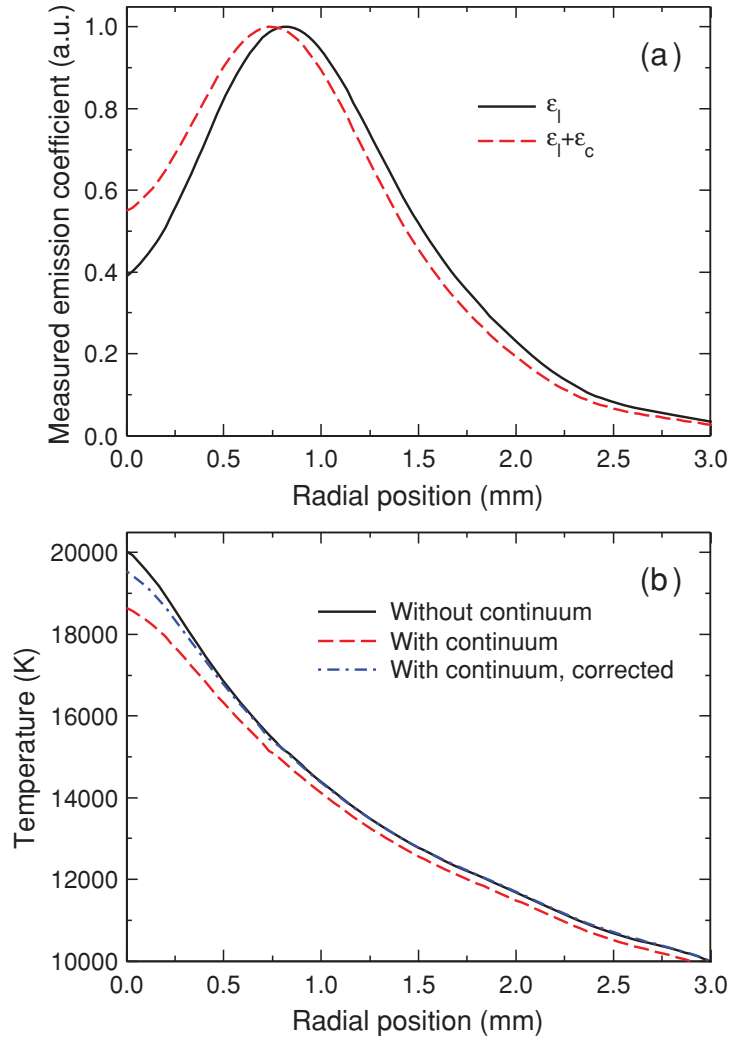

FIG. 5. (Color online) (a) Radial profiles of the normalized emission coefficients for the line and the sum of the line and continuum radiation. (b) Plasma temperature distributions derived from the spectral line emission coefficients.

perature. Figure 5(b) also shows the temperatures corrected the influence of continuum radiation by using the proposed technique as described in Sec. II C. The results are in agreement with these obtained from the line emission coefficients. For temperatures higher than $18000 \mathrm{~K}$, there are minor differences within $500 \mathrm{~K}$. The difference is possibly caused by several factors, such as the errors in Abel inversion, the uncertainties of the Biberman factors, and the accuracies of the measured continuum radiation intensities.

To find which factor is the dominant one and to further check the performance of the proposed technique, we deduced at each arc axial position the temperature differences between the temperatures derived from the emission coefficient profiles with and without subtracting the corresponding continuum radiations. We also calculated the temperature error due to ignoring the continuum radiation as a function of plasma temperature and compared it in Fig. 6 to those from the experiment. When using $\xi_{1}=1.9$ and $\xi_{2}=1.6$, the calculated temperature error is systematically lower than the measurements in the whole temperature range. The nonuniform distribution of the experimental results is indeed due to the errors in Abel inversion. But for temperatures lower than $16000 \mathrm{~K}$, the uncertainties due to Abel inversion will be very small and can be neglected. Therefore, there are other factors which are more important. In Fig. 6 we also show the calculated temperature errors by increasing the values of $\xi_{1}$ and $\xi_{2}$ by a factor of 1.3 and 1.5 , respectively. The calculated errors are in good agreement with the experimental data. Since $\xi_{1}=2.5$ is still in the 


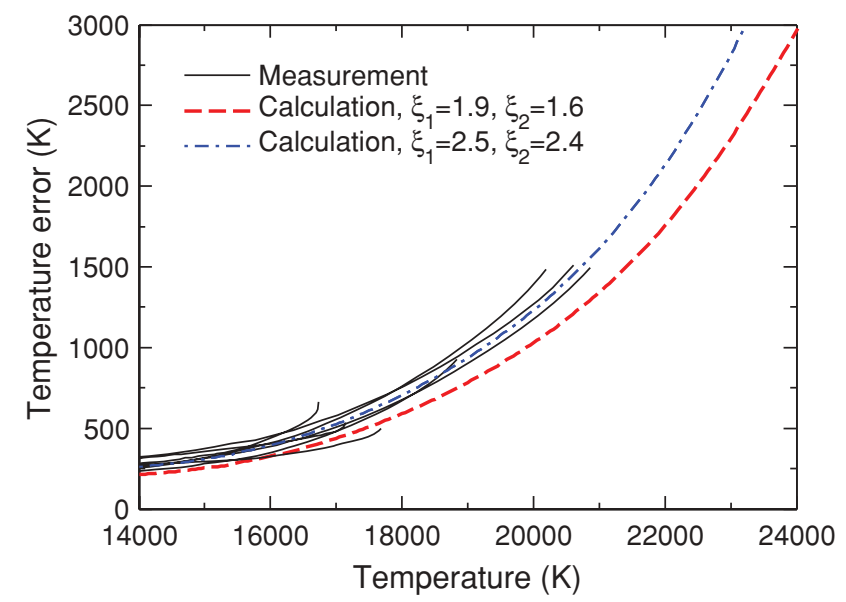

FIG. 6. (Color online) Comparison of the calculated temperature decrease values and the experimentally determined temperature errors at different arc axial positions from 0.5 to $2 \mathrm{~mm}$. A wavelength range of $3.27 \mathrm{~nm}$ was used for the continuum radiation.

uncertainty range of the experimentally measured Biberman factor values, ${ }^{13,19-21}$ it is difficult to tell whether the temperature error differences are mainly caused by uncertainties in the Biberman factors or other sources. It should be noticed that in our measurement we used a spectrograph with a wavelength resolution of $0.156 \mathrm{~nm}$, thus the measured continuum radiation is possibly affected by the line broadening and contamination. This will also increase the temperature differences between the experimental results and the calculations.

Based on the experimental results and analysis, we can see that the Biberman factor $\xi_{1}$ near $696.5 \mathrm{~nm}$ should be close or less than 2.5 , while the value of $\xi_{2}$ is difficult to be experimentally estimated due to the low temperature of the plasma $(<22000 \mathrm{~K})$. Except the errors in the measurements and Abel inversion, which will also exist in other spectroscopic methods, the error caused by the uncertainties of Biberman factors is relatively small. Therefore, the proposed method will give reliable results for plasma temperature measurements.

\section{DISCUSSION}

As described in Sec. II C, the new technique is proposed to determine the plasma temperatures without the measurement of continuum radiation. Although the accuracy of the proposed technique is mainly dependent on the measured spectral line intensities, it is still necessary to analyze the influence of continuum radiation on the results. Particularly, the errors in the measured temperatures caused by the uncertainties of $\xi_{1}$ and $\xi_{2}$ should be presented.

Figure 7 shows the plasma temperature differences calculated by applying the Fowler-Milne method with and without subtracting the continuum radiation from the total emission coefficient. In the calculation, we use $\xi_{1}=1.9$ and $\xi_{2}=1.6$. The error caused by the continuum radiation (including the doubly ionized emission coefficient) becomes larger as plasma temperature increases. For temperatures below $17000 \mathrm{~K}$, the error is relatively small, less than $500 \mathrm{~K}$. For higher temperatures, however, the error increases almost in an

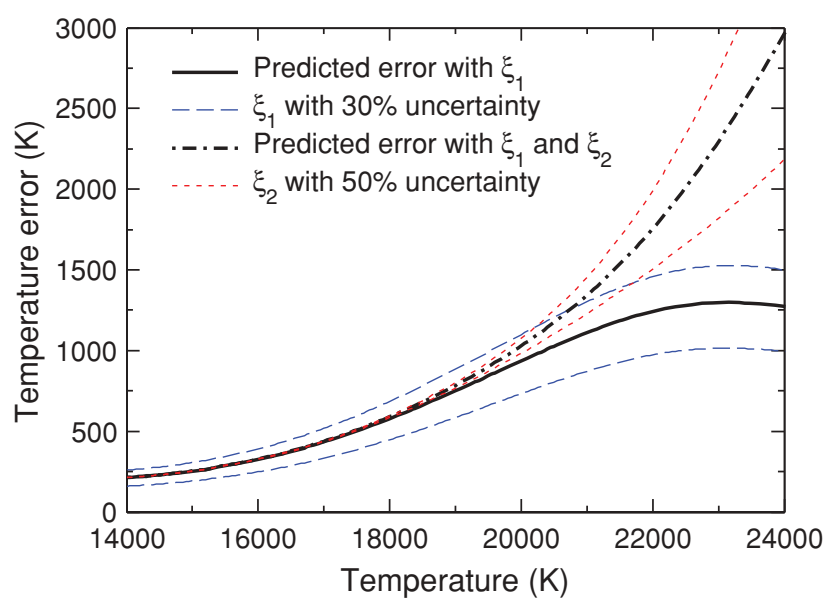

FIG. 7. (Color online) Temperature errors caused by the continuum radiation and the uncertainties in $\xi_{1}$ and $\xi_{2}$ as a function of plasma temperature.

exponential form. For example, the error is only about $1000 \mathrm{~K}$ at $20000 \mathrm{~K}$, but increases to about $3000 \mathrm{~K}$ at $24000 \mathrm{~K}$. Such error indeed cannot be neglected.

Figure 7 also shows the errors caused by $\xi_{1}, \xi_{2}$, and their uncertainties. The error caused by $\xi_{1}$ is less than $300 \mathrm{~K}$ for temperatures below $15000 \mathrm{~K}$ and reaches to a maximum value of about $1300 \mathrm{~K}$ at $23000 \mathrm{~K}$. The error caused by $\xi_{2}$ is measurable only for temperatures higher than $20000 \mathrm{~K}$ but increases much faster. Uncertainties in $\xi_{1}$ and $\xi_{2}$ cause minor errors in the measured plasma temperatures. A $30 \%$ increase in $\xi_{1}$ leads to only about a $250 \mathrm{~K}$ difference at $22000 \mathrm{~K}$, and a $50 \%$ increase in $\xi_{2}$ makes almost the same difference at the same temperature, while the error caused by ignoring the continuum radiation is more than $1750 \mathrm{~K}$.

The different influences of $\xi_{1}$ and $\xi_{2}$ on the measured plasma temperatures can be ascribed to the different contributions of the continuum radiation as a function of plasma temperature. As shown in Fig. 8, the free-free and free-bound radiation from interactions of electrons with singly ionized gases is less than about $40 \%$ of the total emission coefficient for temperatures below $23000 \mathrm{~K}$ and has a similar distribution as the line emission coefficient for higher temperatures, while the radiation from interactions of electrons with doubly ionized gases increases rapidly with temperature and becomes comparable with the line emission coefficient at $24000 \mathrm{~K}$. It is, therefore, not surprise that the error caused by $\xi_{1}$ is limited to a maximum of about $1300 \mathrm{~K}$, and the error caused by $\xi_{2}$ has a steep rise for temperatures higher than $21000 \mathrm{~K}$.

Since most argon plasmas have a temperature less than $22000 \mathrm{~K},{ }^{2,3,5-8}$ only the temperature-averaged value of $\xi_{1}$ is important; the uncertainty in $\xi_{2}$ almost does not affect the accuracy of the proposed technique. For an atomic spectral line with a stronger intensity than that of the Ar I $696.5 \mathrm{~nm}$ line, the contribution of the continuum radiation is much smaller, and thus the continuum radiation related error will decrease further. If the Biberman factors can be determined more accurately, theoretically we will obtain the error-free plasma temperatures, and compared to the Fowler-Milne method, in practice the modified technique should give more reliable 


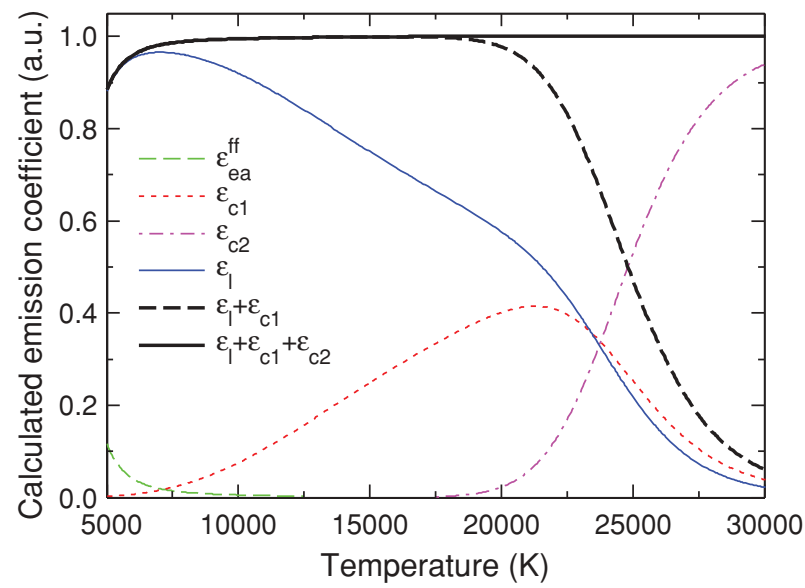

FIG. 8. (Color online) Calculated plasma emission coefficient and its different contributions at $696.5 \mathrm{~nm}$ for an argon arc at atmospheric pressure. All the curves have been normalized with respect to the total emission coefficient distribution.

results as the continuum radiation is difficult to be measured accurately.

The proposed technique is very suitable for measurement of plasma temperatures with a filter-detector imaging system. In this system the bandwidth of the filter is an important parameter, which should be considered in practice. In order to investigate the effect of filter bandwidths on measurement results, the filter transmittance curve was assumed to be a Gaussian function:

$$
f(\lambda)=\exp \left[-4 \ln 2\left(\frac{\lambda-\lambda_{c}}{B}\right)^{2}\right],
$$

where $\lambda_{c}$ is the wavelength of the peak position and $B$ is the full width at half maximum of the filter.

In Fig. 9 the temperature errors at $21000 \mathrm{~K}$ caused by continuum radiation as a function of wavelength shift for the filter with different bandwidths are presented. As the filter bandwidth decreases, the amount of continuum radiation also decreases, and the error in the measured temperature becomes smaller. It seems that the filter with a much narrow bandwidth

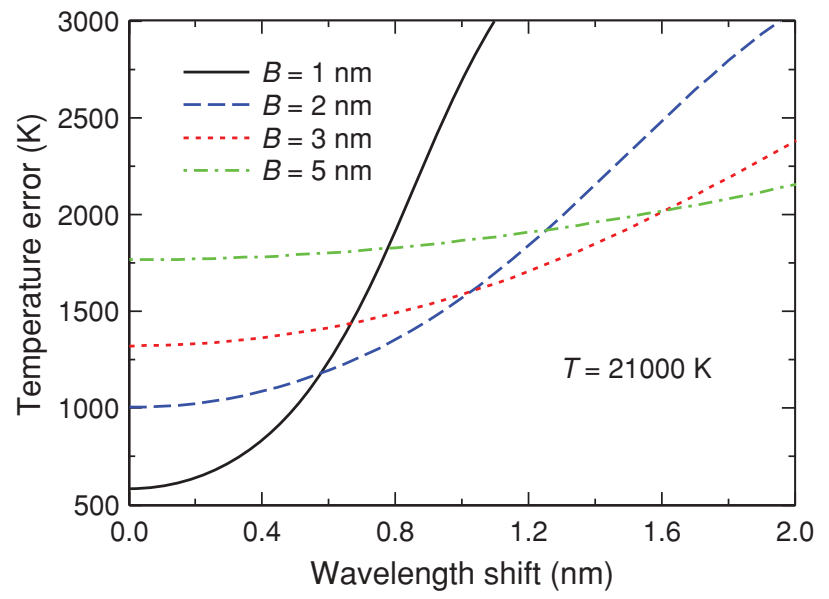

FIG. 9. (Color online) Temperature errors at $21000 \mathrm{~K}$ caused by the continuum radiation as a function of wavelength shift for the filter with different full width at half maximum bandwidths. will be more appropriate, but as the wavelength shift increases the error increases more rapidly for smaller filter bandwidths. Also considering the fact that filters with a bandwidth less than $1 \mathrm{~nm}$ are much expensive and need additional apparatus to prevent drift in the passband, a filter with a bandwidth of between 1 and $3 \mathrm{~nm}$ would be a good choice when using the proposed method to calculate the plasma temperature without the measurement of continuum radiation.

\section{CONCLUSIONS}

For the determination of plasma temperatures using spectroscopic techniques, such as the Fowler-Milne method, the continuum radiation should be subtracted from the measured total emission coefficients to remove its influence on the results. Considering the measurement of continuum radiation, in some cases, is not easy and will complicate the experiments, we have presented a technique for the determination of plasma temperatures without measuring the continuum radiation. The technique takes account all forms of the plasma radiations into the theoretical emission coefficient to avoid the error caused by continuum radiation. Since the amount of continuum radiation is small relative to the line emission coefficient, the technique preserves the advantages of the FowlerMilne method, such as no requirement of absolute intensity calibration, insensitivity to self-absorption of atomic lines, and measurement stability for temperatures between 10000 and $27000 \mathrm{~K}$.

Apart from the requirement of a maximum temperature which should be higher than the normal temperature and the restrictions that are also held for most other spectroscopic methods, such as the LTE condition, the main possible limitation in the proposed technique is that the continuum radiation cannot be accurately quantified due to the uncertainties of the Biberman factors. However, theoretical calculations show that such errors are very small $(<300 \mathrm{~K})$ and almost can be neglected, though the errors caused by continuum radiation for the Ar I $696.5 \mathrm{~nm}$ line with a bandwidth of $3.27 \mathrm{~nm}$ will be more than $1500 \mathrm{~K}$ for temperatures higher than $21000 \mathrm{~K}$. The experimental results obtained by this technique are in good agreement with that from the Fowler-Milne method by considering the continuum radiation, indicating the proposed technique is reliable for plasma temperature measurement.

The technique presented for plasma temperature measurement does not need to know the amount of continuum radiation and thus is particularly suitable for the spectroscopic system with a narrow-band filter of about $3 \mathrm{~nm}$ bandwidth and a high-speed detector to characterize the properties of dynamic plasmas. In combination this technique with the imaging system, the time and spatially resolved properties of a pulsed free-burning arc have successfully been characterized, which will be presented in a subsequent paper.

\footnotetext{
${ }^{1}$ P. Fauchais and A. Vardelle, IEEE Trans. Plasma Sci. 25, 1258 (1997).

${ }^{2}$ C. Fanara and L. Vilarinho, Eur. Phys. J. D 28, 241 (2004).

${ }^{3}$ K. Dzierzega, W. Zawadzki, B. Pokrzywka, and S. Pellerin, Phys. Rev. E 74, 026404 (2006).

${ }^{4}$ H. R. Griem, Principles of Plasma Spectroscopy (Cambridge University, Cambridge, England, 1997).
} 
${ }^{5}$ H. N. Olsen, J. Quant. Spectrosc. Radiat. Transf. 3, 305 (1963).

${ }^{6}$ J. Haidar and A. J. D. Farmer, J. Phys. D: Appl. Phys. 27, 555 (1994).

${ }^{7}$ A. B. Murphy, Rev. Sci. Instrum. 65, 3423 (1994).

${ }^{8}$ K. Hiraoka, T. Shiwaku, and T. Ohji, Weld. Int. 11, 688 (1997).

${ }^{9}$ D. Samsonov and J. Goree, IEEE Trans. Plasma Sci. 27, 76 (1999).

${ }^{10}$ M. Takeuchi and T. Kubono, IEEE Trans. Plasma Sci. 28, 991 (2000).

${ }^{11}$ S. Ma, H. Gao, and L. Wu, IEEE Trans. Plasma Sci. 36, 1054 (2008).

${ }^{12}$ S. Ma, H. Gao, L. Wu, and S. Zheng, Meas. Sci. Technol. 19, 105602 (2008).

${ }^{13}$ A. T. M. Wilbers, G. M. W. Kroesen, C. J. Timmermans, and D. C. Schram, J. Quant. Spectrosc. Radiat. Transf. 45, 1 (1991).

${ }^{14}$ J. J. Beulens, M. J. de Graaf, and D. C. Schram, Plasma Sources Sci. Technol. 2, 180 (1993).

${ }^{15}$ B. Pokrzywka, S. Pellerin, K. Musiol, and J. Chapelle, J. Phys. D: Appl. Phys. 32, 1665 (1999).

${ }^{16}$ E. Iordanova, N. de Vries, M. Guillemier, and J. A. M. van der Mullen, J. Phys. D: Appl. Phys. 41, 015208 (2008).

${ }^{17}$ R. S. Devoto, Phys. Fluids 16, 616 (1973).
${ }^{18}$ D. C. Schram, Technische Universiteit Eindhoven, Eindhoven, The Netherlands, private communication (22 September 2009).

${ }^{19}$ A. T. M. Wilbers, G. M. W. Kroesen, C. J. Timmermans, and D. C. Schram, Meas. Sci. Technol. 1, 1326 (1990).

${ }^{20}$ D. Hofsaess, J. Quant. Spectrosc. Radiat. Transfer 19, 339 (1978).

${ }^{21}$ L. G. D’yachkov, Y. K. Kurilenkov, and Y. Vitel, J. Quant. Spectrosc. Radiat. Transf. 59, 53 (1998).

${ }^{22}$ J. Menart and S. Malik, J. Phys. D: Appl. Phys. 35, 867 (2002).

${ }^{23}$ J. Menart, Wright State University, Dayton, OH 45435, USA, private communication (16 July 2006).

${ }^{24}$ G. Kühn, F. Kemanna, and M. Kock, J. Quant. Spectrosc. Radiat. Transf. 105, 102 (2007).

${ }^{25}$ A. B. Murphy, Phys. Rev. Lett. 89, 025002 (2002).

${ }^{26}$ H. R. Griem, Plasma Spectroscopy (McGraw-Hill, New York, 1964).

${ }^{27}$ L. Bober and R. S. Tankin, J. Quant. Spectrosc. Radiat. Transf. 9, 855 (1969).

${ }^{28}$ S. Ma, H. Gao, and L. Wu, Appl. Opt. 47, 1350 (2008). 